\title{
Epimysial Transposition Flap: A Novel Technique for Coverage of Exposed Tendon after Tissue Transfer
}

\author{
Nicholas F. Lombana, MD ${ }^{1}$ Reuben A. Falola, MD, MPH ${ }^{1} \quad$ Michael Scott, BS ${ }^{1}$ \\ Michel H. Saint-Cyr, MD, FRCS ${ }^{1}$
}

${ }^{1}$ Division of Plastic and Reconstructive Surgery, Baylor-Scott and White, Temple, Texas

Address for correspondence Michel H. Saint-Cyr, MD, FRCS, Division of Plastic and Reconstructive Surgery, Baylor-Scott and White, 2401

J Reconstr Microsurg Open 2021;6:e51-e56.

South 31st Street, Temple, Texas 76508 (e-mail: msaintcyr@me.com).

\begin{abstract}
Background The radial forearm flap (RFF) is a versatile flap that can be used for softtissue coverage or as a source of vascularized bone during reconstruction. A problem that can arise after RFF harvest is tendon exposure in the donor site, which may lead to tendon injury and restriction of the hand and wrist's range of motion. Many different methods for providing adequate tendon coverage in the donor site after RFF harvest have been reported. We present a novel technique for coverage of exposed tendon in the volar forearm with an epimysial transposition flap and a split-thickness skin graft (STSG).

Methods Retrospective review of two cases: one salvage case with exposed tendon after harvesting an RFF and another with exposed volar forearm tendon after traumatic injuries. Variables of interest were post-operative STSG integration and hand/wrist range of motion.

Keywords

- radial forearm flap

- epimysial transposition flap

- upper extremity reconstruction

Results Both cases demonstrated $100 \%$ graft take at 5 -week follow-up. No decrease in hand/wrist range of motion in the patient that could undergo testing.

Conclusion We introduce two cases for coverage of exposed flexor tendon in the volar forearm with an epimysial transposition flap. This can be used as a salvage operation for coverage of exposed tendon or as an acute method of tendon coverage prior to skin grafting. In both cases, the skin graft had excellent incorporation.
\end{abstract}

The radial forearm flap (RFF) is used in various reconstructive procedures in the head and neck as a free flap as well as in the upper extremities as a pedicled flap. This flap has a range of therapeutic uses, including soft tissue coverage and vascularized tendon and/or bone reconstruction, if necessary. One of the problems that can arise when using the radial forearm flap for soft tissue coverage is tendon exposure within the

received

July 17,2020

accepted after revision

January 27, 2021
DOI https://doi.org/ $10.1055 / \mathrm{s}-0041-1729954$. ISSN 2377-0813. donor site after tissue transfer. Exposed tendon is particularly problematic as it leads to tendon desiccation, functional range of motion (ROM) restrictions, tendon adhesions, subsequent reepithelization, and loss of extracellular matrix., ${ }^{1,2}$ Major contraindications to the radial forearm flap are inadequate collateral blood flow to the hand via the ulnar artery, arterial anomalies, and prior arterial injury.

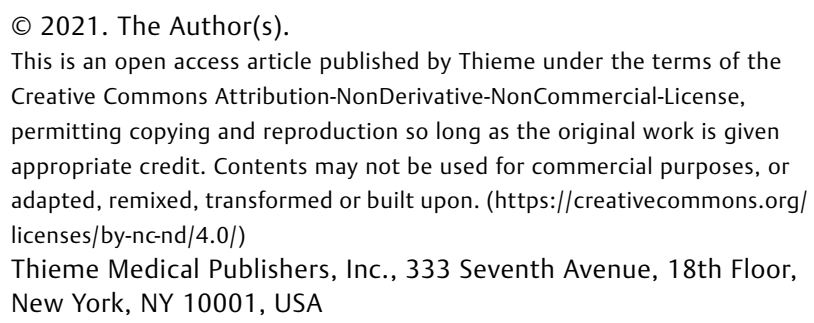


Due to the negative outcomes associated with tendon exposure after tissue transfer, many different methods have been proposed for attaining adequate tendon coverage. In this report, we present a novel technique for coverage of exposed tendon with an epimysial transposition flap in the donor site after tissue transfer of a pedicled radial forearm flap as a salvage operation and another case of its use for acute coverage of exposed tendon.

\section{Case Presentation 1: Epimysial Transposition Flap in Radial Forearm Flap Donor Site}

A 34-year-old Hispanic male suffered a fracture of his left olecranon without displacement. The patient was initially treated with closed reduction and external fixation. He was subsequently taken to the operating room for excision and debridement (E\&D) of the left olecranon and open reduction with internal fixation (ORIF). The patient underwent two further E\&Ds of the left olecranon with application of negative pressure wound therapy due to chronic wounds and exposed hardware in the beginning of August 2019. Five days after his last E\&D, he was taken to the operating room by plastic and reconstructive surgery for definitive coverage of the left olecranon with a pedicled left radial forearm flap.

Preoperatively, a Doppler Allen's test was performed bilaterally, demonstrating equal ulnar and radial dominance of the left upper extremity and patency of the palmar arch with radial occlusion. The left elbow wound was initially debrided sharply of skin, subcutaneous fat, and granulation tissue. The area of debridement measured $2 \mathrm{~cm} \times 10 \mathrm{~cm}$ and the total defect measured $6 \mathrm{~cm} \times 12 \mathrm{~cm}$. Measurement of the tissue necessary for complete coverage of the defect was done and initial incision was begun distally near the wrist crease to identify the radial artery. Once the radial artery was ligated, incision was done along the remainder of the flap along the markings. Dissection began in the ulnar-to-radial direction in the suprafacial plane until the septum overlying the radial artery, which protruded between the brachioradialis muscle belly and the flexor carpi radialis (FCR) tendons, was identified. At this point, dissection was begun on the opposite side of the flap, moving in a radial-to-ulnar direction suprafacially, until the septum was encountered (-Fig. 1A, B). Once the pedicle was identified, dissection was begun distal to proximal until the vessel branchpoints near the antecubital fossa were encountered ( $\mathbf{- F i g . ~ 1 C , ~ D ) . ~}$ After the flap was completely elevated ( - Fig. 1E), a subcutaneous tunnel was made for flap insertion into the defect (-Fig. 1F). The flap was inset with 3-0 monocryl deep dermal and 3-0 prolene vertical mattress sutures and the donor site was covered with Integra. Upon closure of the recipient site, the flap was warm, pink, with a strong arterial signal on Doppler (-Fig. 2).

The proximal donor site was closed in a curvilinear fashion with advancement of radial skin to cover the cephalic vein and superficial sensory branch of the radial nerve. At this point, the remaining defect measured $13 \mathrm{~cm} \times 6 \mathrm{~cm}$ with exposed tendon. Due to its great safety profile, and ease of use, Integra was placed over the donor site with plans for skin grafting at a later stage after incorporation.

\section{Surgical Technique}

One month later, the patient returned to the operating room (OR) for placement of a split thickness skin graft (STSG) over the left volar forearm donor site. It was decided intraoperatively to debride the Integra and subcutaneous tissue, with a final wound dimension of $13 \mathrm{~cm} \times 7 \mathrm{~cm}$. Postdebridement,
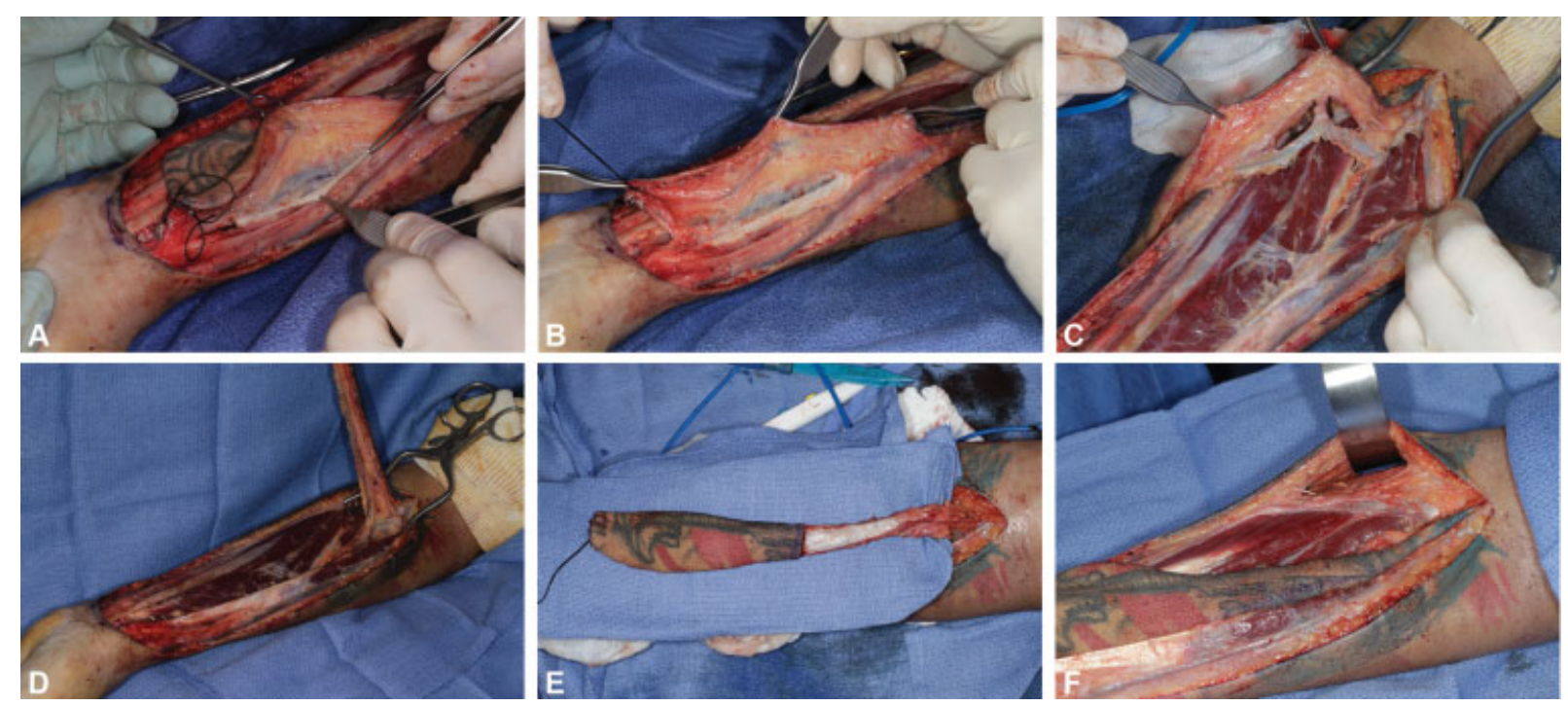

Fig. 1 Volar view of the left forearm. (A, B) The radial artery is ligated, and the dissection is performed in the suprafacial plane. (C, D) Once the pedicle was identified, dissection was begun distal-to-proximal until the vessel branchpoints near the Antecubital fossa were encountered. (E) The radial forearm flap is completely elevated. (F) A subcutaneous tunnel was made for flap insertion into the defect. 


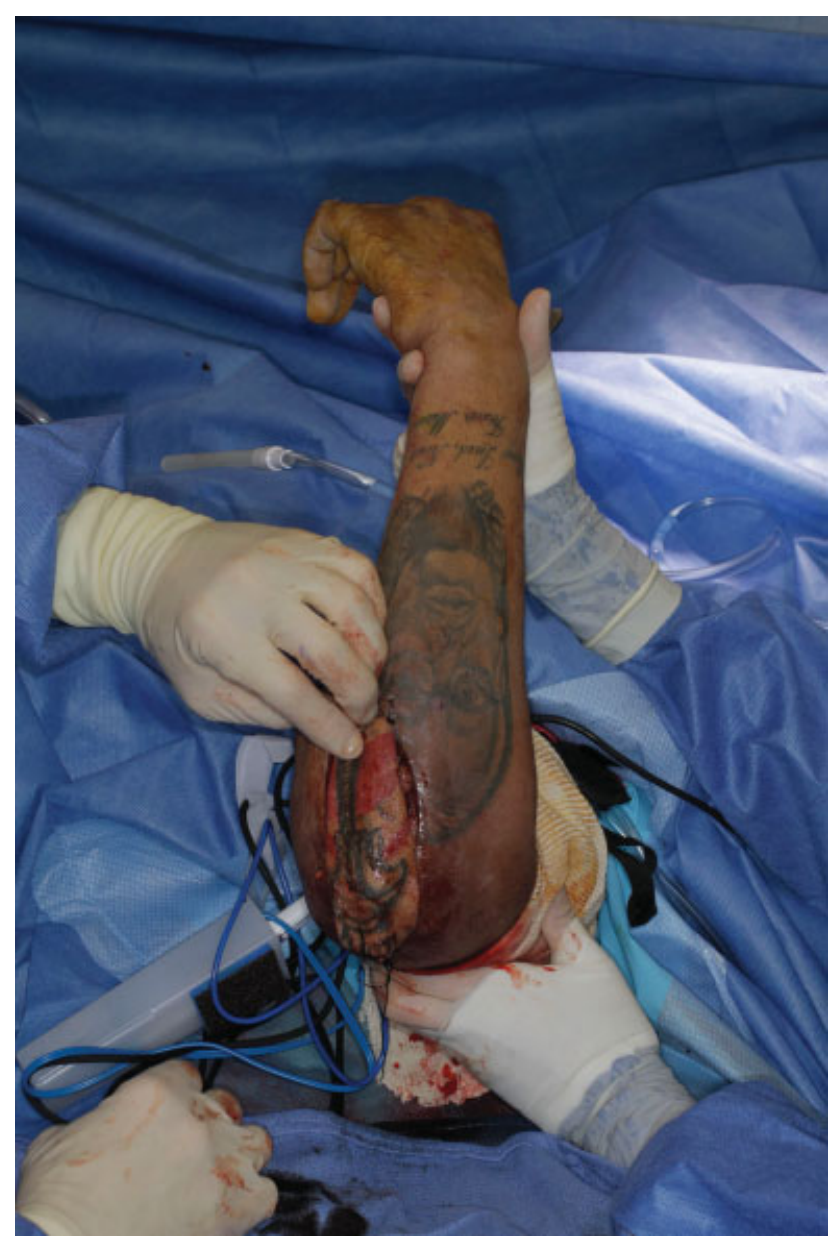

Fig. 2 Dorsal view of the flexed left olecranon. The flap has been tunneled from the volar to the dorsal forearm and inset with 3-0 monocryl deep dermal and 3-0 prolene vertical mattress sutures. The flap was warm, pink, with a Dopplerable arterial signal. exposed FCR and palmaris longus (PL) tendons were visible. Tenolysis was performed to release the exposed tendons from surrounding scar tissue. Once sufficient tenolysis was performed, two epimysial transposition flaps were made to cover the FCR and PL ( - Fig. 3). The PL was elevated to expose the FDS muscle belly. At this point, the FDS muscle belly was incised to create an epimysial flap that was reflected radially. The PL was then laid onto the remaining muscle and the new epimysial flap was transposed over the PL and sutured with 4-0 chromic. Radially, the FCR was elevated to expose the underlying FDS muscle belly. Again, the muscle belly was incised and an epimysial transposition flap was created. The FCR was then laid onto the remaining FDS muscle belly and the new epimysial transposition flap was used to cover the exposed FCR. After coverage of the exposed tendons was complete, a STSG was harvested from the left thigh and sutured onto place in the left volar forearm.

\section{Follow-up}

Due to the coronavirus (COVID-19) pandemic and the patient living more than 6 hours from the hospital, a telemedicine appointment was scheduled to assess the patient's ROM. At 6-month follow-up of both donor and recipient sites, sites are well-healed with adequate soft tissue coverage and preserved range of motion (-Fig. $\mathbf{4}$; - Video $\mathbf{1}$ ). The patient has gone back to work and has assumed his premorbid, presurgical functional state, endorsing complete ROM with flexion and extension of the digits, wrist, and elbow. The patient endorses no major limitations but has experienced mild hyperesthesia in the recipient site. The patient wears long sleeves as a protective measure. He denies any selfimage concerns as a result of surgery and is happy with the aesthetic and functional outcome of his reconstruction.
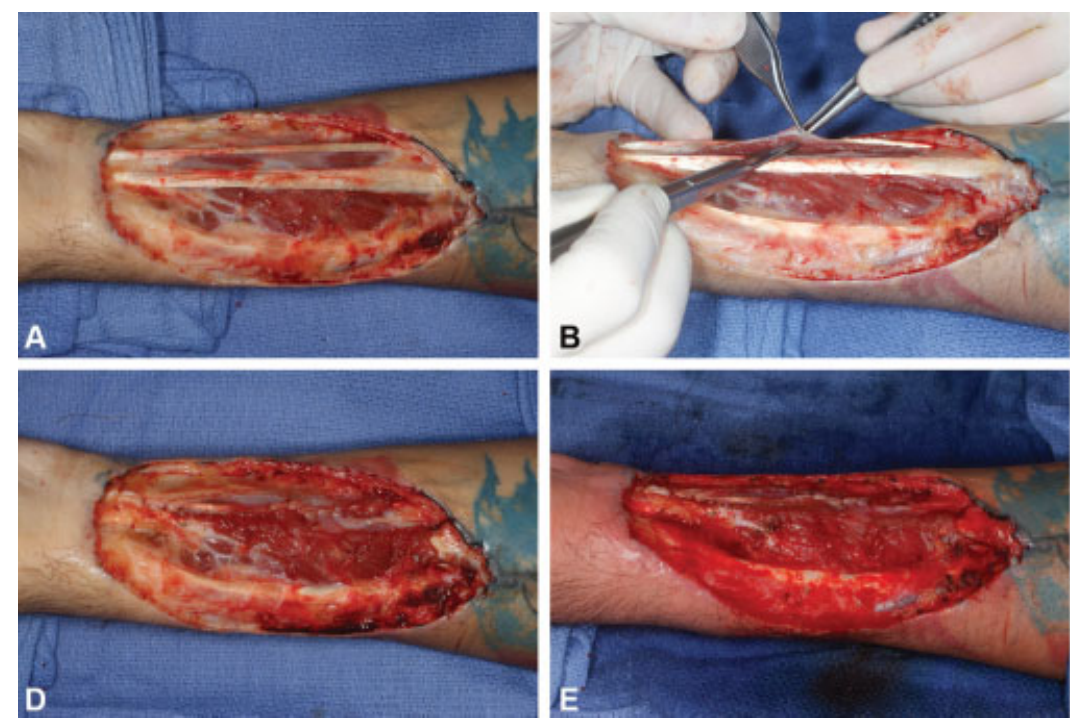
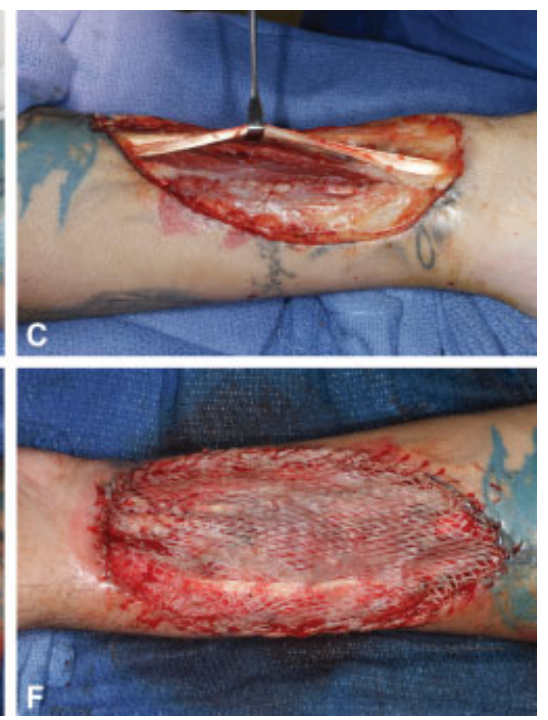

Fig. 3 (A) Volar view of exposed flexor carpi radialis (FCR) and palmaris longus (PL) tendon of left forearm, post-debridement. (B, C) The surrounding scar tissue was excised from the exposed tendons. (D) Epimysial transposition flaps are used to cover the tendons and sutured with 4-0 chromic. (E, F) The tourniquet was released and a split thickness skin graft (STSG) was harvested from the left thigh and sutured into place. 

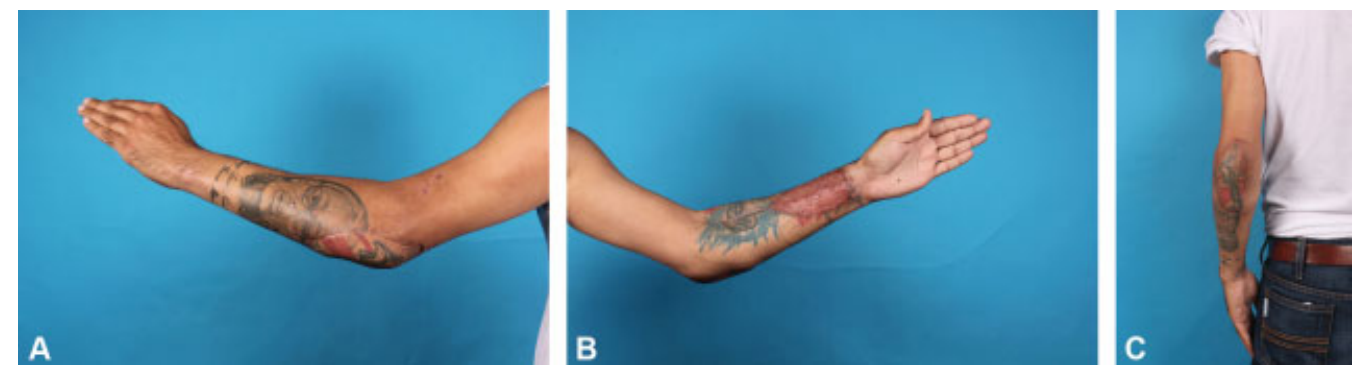

Fig. 4 (A-C) At follow-up, 6 months after surgery, both donor and recipient sites are well-healed with adequate soft tissue coverage and preserved range of motion.

\section{Video 1}

The 6-month follow-up demonstrating intact arm flexion and extension, wrist ulnar flexion, and extension followed by wrist flexion without ulnar flexion (volar view), digital flexion, and extension (dorsal view), and wrist flexion and extension (dorsal view). Online content including video sequences viewable at: https://www.thieme-connect.com/products/ ejournals/html/10.1055/s-0041-1729954.

\section{Case Presentation 2: Epimysial Transposition Flap for Acute Coverage of Exposed Tendon}

A 39-year-old male who suffered multiple bilateral metacarpal and phalanx fractures with soft tissue injuries of bilateral upper extremities due to a motorcycle collision in early June 2020. Among the soft tissue injuries was an $11-\mathrm{cm}$ right volar forearm laceration. This laceration was used by orthopaedic surgery to reduce a shaft fracture of the radius and ulna. After reduction, primary closure could not be obtained without risking muscular compromise and ischemia, therefore a wound VAC was applied. Two days later, the patient was taken back to the OR for debridement and attempted closure of the right volar forearm incision, which measured $8 \mathrm{~cm} \times 3 \mathrm{~cm} \times 4 \mathrm{~cm}$ after debridement and sequential closure (-Fig. 5A, B). Seven days after debridement of the right volar forearm wound, he was taken back to the operation room by plastic and reconstructive surgery for soft tissue coverage and reduction of the multiple right-hand fractures.

After a combination of closed and open reduction with pinning was used on the right-hand fractures, attention was turned to the right volar forearm wound. The negative pressure wound dressing was removed, revealing an exposed flexor carpi radialis (FCR) at the base of the wound. Tenolysis of the FCR was performed and an epimysial transposition flap of the underlying muscle belly was created and transposed over the exposed FCR tendon. The flap was then secured into place with 2-0 vicryl in a figure-of-eight fashion. After measurement of the forearm defect, a $45 \mathrm{~cm}^{2}$ pie crusted STSG was harvested from the right thigh and sutured into the recipient site with 4-0 chromic.

At 5-week follow-up, the patient had $100 \%$ graft take to the right volar forearm (-Fig. $\mathbf{6}$ ). Due to the multiple digit fractures of bilateral hands requiring reduction and fixation, the patient has undergone multiple session with occupational therapy. On physical examination, he had limited flexion and extension of the right wrist due to stiffness, he had full extension of all digits and limited flexion of the right little, ring, and long fingers secondary to stiffness. He was taken back to the operating room 3 months after the initial surgery due to persistent subluxation of the right long finger at the distal interphalangeal joint. Due to the timing of this patient's injury, he is still in a cast and follow-up ROM pictures and exercises have not been able to be obtained at the time of manuscript writing.
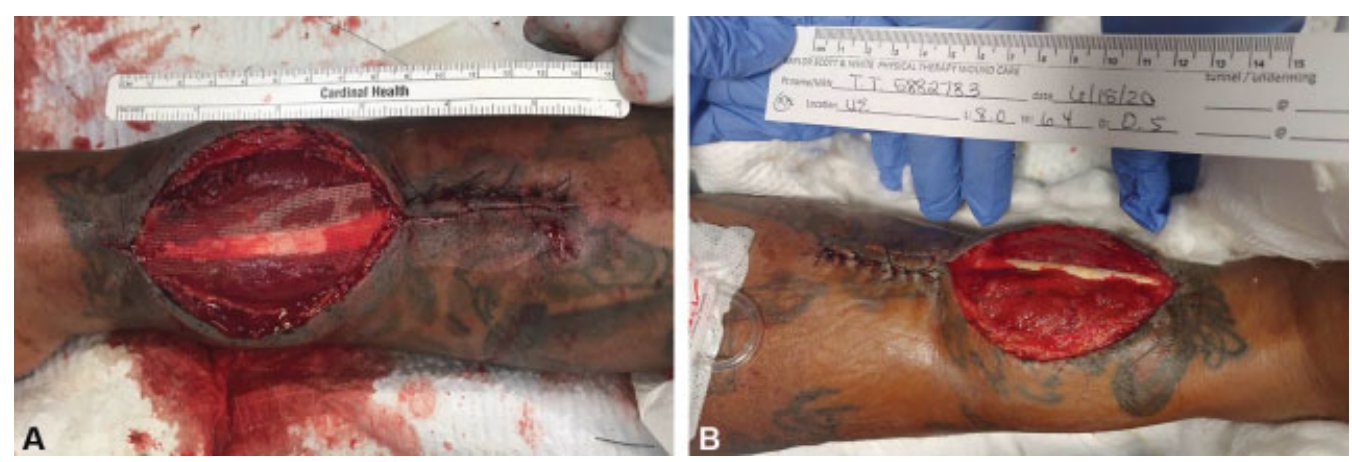

Fig. 5 Volar view of right forearm. (A) Right volar forearm wound immediately after debridement. (B) Right volar forearm wound 4 days after debridement and negative pressure wound therapy. 


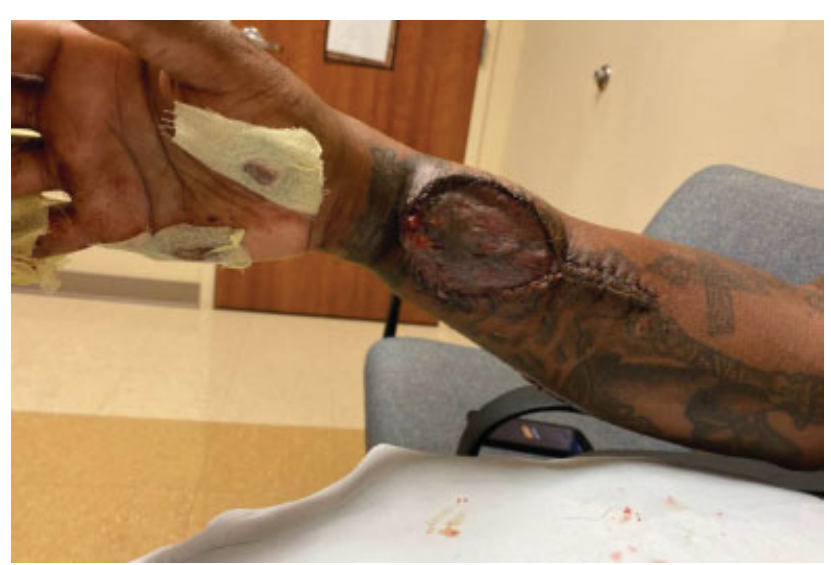

Fig. 6 Right volar forearm STSG demonstrating 100\% take at 5 weeks postoperatively. STSG, split thickness skin graft.

\section{Discussion}

The objective of this report is to introduce a novel technique for coverage of exposed flexor tendons in the volar forearm with an epimysial transposition flap. In this report, we present two cases of its use, as a salvage operation for coverage of exposed tendon after failure of Integra and as an acute coverage of exposed tendon for a STSG bed. As a workhorse flap for upper extremity reconstruction and head and neck reconstruction, the radial forearm flap has an immense role in reconstructive plastic surgery. ${ }^{3}$ Despite the many uses of this flap and the positive outcomes associated with its use, many plastic and reconstructive surgeons struggle with the postoperative donor site morbidity that many patients experience after undergoing reconstruction. Many of the effects that patients experience after a radial forearm flap have to do with functional outcomes, such as decreased wrist mobility, hand strength, and sensibility. ${ }^{4}$ Another common complication that reconstructive plastic surgeons encounter after harvesting this flap is exposure of the underlying tendons in the forearm.

The normal anatomy of a tendon includes a paratenon with a rich blood supply surrounded by synovial fluid that allows for proper gliding of the tendon and diffusion of nutrients required to sustain the tendon. ${ }^{2}$ Without this paratenon, tendon exposure leads to a risk of desiccation, infection, adhesion to surrounding structures, and scar formation. ${ }^{5,6}$ Furthermore, exposed tendons often lose the ability to glide against smooth surfaces which may lead to diminished joint function and mobility, as well as decreased wrist mobility and hand strength. ${ }^{2,4}$ Finally, delayed wound healing may result in wounds with exposed tendons, as the resultant adhesions and scar tissue that result from tendon desiccation can interrupt proper nutrition delivery to the tendon. $^{2}$

Due to these challenges, many surgical techniques have been developed and discussed to decrease donor-site morbidity and improve postoperative outcomes for patients. In 2018, Pabst et $\mathrm{al}^{3}$ analyzed 39 studies in the MEDLINE database and discovered four main method for closure of the donor site of a radial forearm flap: full-thickness skin graft (FTSG), STSG, closure by local flaps, and other methods such as allografts, tissue expanders, and negative-pressure wound therapy (NPWT). The authors also found that the most common side effects were poor wound healing due to exposed tendons. Similarly, in 2018, Etzkorn et al ${ }^{7}$ presented a case in which a single-lobed transposition flap was used to cover exposed tendons on the dorsal hand. They found the following three benefits to the use of transposition flaps and skin grafts in this context: (1) transposition flaps are a great way to cover exposed tendons due to their robust vascularity, (2) closure of the donor site with a skin graft decreases closure tension, and, finally, (3) combination of skin grafts and transpositions flaps are a reliable and time-effective method for single-stage reconstruction.

As mentioned above, donor-site postoperative mobility is a challenge that many reconstructive surgeons face after harvesting a radial forearm flap. In 2003, Skoner et $\mathrm{al}^{8}$ retrospectively reviewed 12 patients who had undergone a radial forearm free flap for head and neck reconstruction with skin grafting for closure of the donor site to analyze short-term functional donor site deficiencies. When comparing preoperative versus postoperative functionality, they found statistically significant decreases in forearm supination and protonation, as well as wrist flexion and extension. These results show that skin grafting alone of the donor site may not be enough to prevent donor site morbidity. Perhaps most applicable to our technique for coverage of exposed tendon, a study by Jaquet et $\mathrm{al}^{9}$ was published in 2012. The authors examined 22 patients who had received a STSG alone and 22 patients who had received an ulnar-based transposition flap (UBTG) for coverage of the donor site in a RFF with exposed tendon. They found that wrist extension and overall wrist range of motion were better in the UBTF group than in the STSG group.

In case 1 , Integra was the initial choice of the surgeon for coverage of the exposed tendon in preparation for skin grafting. Some of the Integra had not incorporated well along with some tissue, and had to be debridement in preparation for skin grafting, leaving new areas of exposed tendon. This was when an epimysial transposition flap was considered as a salvage operation, so as not to delay skin grafting. The studies mentioned so far support the use of a local epimysial transposition flap for coverage of exposed tendon followed by STSG for final closure of the donor site defect. At 6 months, our patient demonstrated intact arm flexion and extension, ulnar wrist flexion, and extension followed by wrist flexion without ulnar flexion and digital flexion and extension.

In case 2, the patient had exposed FCR and the decision was made to use the epimysial transposition flap acutely to provide coverage for the exposed tendon and creation of a viable wound bed onto which to graft a STSG. Due to the recent timing of the patient's trauma and stiffness from being splinted postoperatively, the full ROM of the FCR could not be fully evaluated. The STSG did have excellent take at 5week postoperatively, demonstrating the epimysial transposition flap to be a viable recipient site for a skin graft.

Another common option for treatment of exposed tendon is the placement of a NPWT device which promotes the 
development of granulation tissue over exposed tendons. However, NPWT can take longer, is more costly, and may cause more stiffness in the long run when compared with the epimysial flap. An epimysial transposition flap provides a safe environment in which tendons can theoretically slide with minimal obstruction and thus decrease postoperative mobility issues. In addition, coverage of exposed tendons in this way may prevent the complications that are commonly associated with exposed tendons such as adhesion and scar formation surrounding the tendons. Although our case report demonstrates minimal functional postoperative morbidity at 6 months, further research should focus on long-term functional outcomes among multiple patients. Finally, the use of a split-thickness skin graft to close the donor site can minimize the morbidity of requiring another flap and further surgery, while assuring a tension-free closure.

\section{Conclusion}

The radial forearm flap has been a workhorse flap for the reconstructive plastic surgeon and will continue to be for many years to come with diverse uses for upper extremity and head and neck reconstruction. Despite its success as a reconstructive tool, surgeons must find ways to deal with the postoperative donor site morbidity that patients' occasionally experience. In this paper, we have presented a technique for adequate coverage of exposed forearm flexor tendons. We used an epimysial transposition flap as a salvage operation to cover exposed tendons followed by placement of a STSG over the defect. We also demonstrated that this technique can be used in the acute phase to cover exposed tendon. We believe that this operation for coverage of exposed tendon is an essential tool for the reconstructive surgeon to have in their armamentarium, due to ease of the technique, decreased recovery time, and improved postoperative mobility.

Conflict of Interest

None declared.

\section{References}

1 Simman R, Hermans MHE. Managing wounds with exposed bone and tendon with an esterified hyaluronic acid matrix (eHAM): a literature review and personal experience. J Am Coll Clin Wound Spec 2018;9(1-3):1-9

2 Trumble TE. Principles of Hand Surgery and Therapy. Philadelphia, PA: W.B. Saunders; 2000

3 Pabst AM, Werkmeister R, Steegmann J, Hölzle F, Bartella A. Is there an ideal way to close the donor site of radial forearm free flaps? Br J Oral Maxillofac Surg 2018;56(06):444-452

4 Nehrer-Tairych GV, Millesi W, Schuhfried O, Rath T. A comparison of the donor-site morbidity after using the prelaminated fasciomucosal flap and the fasciocutaneous radial forearm flap for intraoral reconstruction. Br J Plast Surg 2002;55(03):198-202

5 Bucholz RW, Heckman JD, Court-Brown CM, Rockwood CA, Green DP, eds. Rockwood and Green's Fractures in Adults. Vol. 1;Philadelphia, PA: Lippincott, Williams \& Wilkins; 2006

6 Davis-Zeek D. Pearls for practice: managing wounds with exposed tendon. Wound Management and Prevention 2009;55(10):

7 Etzkorn JR, Sobanko JF, Shin TM, Miller CJ. Repair of a large dorsal hand defect with exposed tendons. Dermatol Surg 2018;44(12): 1583-1586

8 Skoner JM, Bascom DA, Cohen JI, Andersen PE, Wax MK. Short-term functional donor site morbidity after radial forearm fasciocutaneous free flap harvest. Laryngoscope 2003;113(12):2091-2094

9 Jaquet Y, Enepekides DJ, Torgerson C, Higgins KM. Radial forearm free flap donor site morbidity: ulnar-based transposition flap vs split-thickness skin graft. Arch Otolaryngol Head Neck Surg 2012; 138(01):38-43 